\title{
PROTECTION ET VALORISATION DU PATRIMOINE INDUSTRIEL : SIGNES DE RECONNAISSANCE POUR L'ENTREPRISE
}

\author{
Patrice de la Broise ${ }^{1}$
}

\section{Le patrimoine : un concept utile pour penser I'entreprise}

C'est à peine si le concept de communication appliqué à l'entreprise est stabilisé, que d'autres notions lui sont déjà proposées comme prolongements ou substituts. Il en va ainsi de la "culture d'entreprise", comme de "l'identité d'entreprise", dont l'emploi répété reste approximatif. Ces concepts, au demeurant séduisants, ne sont pas mieux maîtrisés et n'éclairent que partiellement la complexité d'une organisation à caractère industriel.

C'est pourquoi nous attirons l'attention sur l'avantage théorique d'un recentrage sur l'entreprise, entendue comme organisation singulière. L'entreprise, en effet, n'est pas une organisation comme les autres. Elle se distingue par la diversité des acteurs qu'elle mobilise : fournisseurs, pouvoirs publics, clients, syndicats, associations de

1 Centre de Recherche sur la Culture et les Musées (CRCM) - Université de Bourgogne. 
consommateurs... Elle diffère, surtout, par son objet (une production marchande) et par sa finalité (la recherche du profit).

Il reste que, dans l'entreprise, du culturel se manifeste qu'on ne voyait pas auparavant. Comment le nommer ? Comment approcher la dimension culturelle de l'entreprise, sans occulter son projet économique ? Pour bousculer cette dialectique, nous proposons de recourir à un concept suffisamment proche de la question économique et du champ culturel pour être pertinent : celui de patrimoine.

Cela exige, d'abord, de revenir à la définition de ce concept et d'en identifier les formes particulières appliquées à l'entreprise. Du plus tangible au plus immatériel, du plus ancien au plus contemporain, le patrimoine recouvre un ensemble contrasté et inégalement partagé de signes à caractère historique, technique, esthétique... A défaut d'en dresser l'inventaire exhaustif, nous préciserons les objectifs assignés à leur valorisation. Nous verrons notamment comment la patrimonialisation s'opère diversement selon les stratégies et les moyens privilégiés par l'entreprise.

Sous l'angle de la communication, nous montrerons principalement par quels atouts et dans quelles limites le patrimoine de l'entreprise peut enrichir une politique de communication institutionnelle, comment le patrimoine invoqué et convoqué par l'entreprise peut contribuer efficacement à sa visibilité sociale et culturelle.

Au-delà, c'est la reconnaissance de l'entreprise comme espace public qui est de nouveau mise en question. La conjonction de l'économique et du culturel confère à l'appareil productif une sociabilité nouvelle. Reste à savoir comment, entre visibilité et transparence, peut s'opérer la rencontre du capital et du social, de l'expert et du profane.

\section{Parcours sémantique et pertinence d'un concept}

Il faut d'abord relever, avec Desvallées, les glissements sémantiques du mot patrimoine qui "non seulement n'est pas récent, mais est bizarrement apparu pour disparaître et réapparaître"l.

Le patrimoine désigne l'ensemble des droits réels cumulés par un titulaire et transmis par dévolution héréditaire. C'est là une définition juridique, de loin antérieure à toute autre acception.

1 A. DesvalléEs, "Émergence et cheminements du mot patrimoine", Musées \& collections publiques de France, $\mathrm{n}^{\circ} 208$, sept. 1996, p. 6-29. 
C'est à la Révolution Française que nous devons l'extension de ce cadre juridique, lorsque celle-ci élève les biens de la Nation au rang de "Patrimoine National". Ainsi s'opère, tardivement, le passage du patrimoine individuel au patrimoine collectif.

Mais pour ce patrimoine fraîchement ré-affecté (nationalisé) les soins se font attendre. Les premiers textes législatifs sur l'intervention des pouvoirs publics datent seulement du XIXe siècle, la plus grande attention étant portée à la sauvegarde des monuments historiques. Progressivement l'ensemble du domaine archéologique et artistique fera l'objet des mêmes sollicitudes.

Il faut, enfin, patienter jusque dans les années 1970, pour que, sous l'impulsion préalable des organismes internationaux, le patrimoine déborde véritablement ce cadre monumental et artistique. C'est donc très récemment que le mot patrimoine "s'applique à des secteurs de plus en plus nombreux, du plus classique et du plus sélectif au plus vernaculaire et au plus immatériel (...) depuis le «patrimoine architectural» en 1973 et le «patrimoine monumental» en 1974, jusqu'au patrimoine archéologique, au patrimoine industriel et au patrimoine régional, en $1976^{1}$.

Ce cheminement historique du mot patrimoine n'aurait qu'un intérêt modeste, s'il ne traduisait une modification sensible du sens attribué à ce concept. Cette évolution s'opère à plusieurs niveaux. En premier lieu, on observe un glissement de la notion de propriété à celle d'appropriation morale ${ }^{2}$. De plus, le patrimoine est progressivement entendu, non plus comme un bien réductible à un individu, mais comme signe de reconnaissance d'une communauté toute entière, susceptible d'en partager l'intérêt.

Par ailleurs, la notion de patrimoine n'est plus seulement circonscrite aux biens reconnus pour leur valeur esthétique ou monumentale. Elle recouvre également d'autres témoignages, matériels ou immatériels. L'élargissement du patrimoine aux formes les plus vernaculaires d'une communauté indique combien celle-ci est prompte à reconnaître une valeur à toute chose, hors des canons esthétiques et/ou intellectuels classiques.

Enfin, et surtout, l'historicité n'est plus une condition nécessaire -et encore moins suffisante- à une patrimonialisation. L'ouverture des musées et expositions aux formes les plus actuelles de l'art, de la

1 Ibidem.

2 Ibidem. 
science, de la technique et de l'industrie modifie sensiblement le rapport au temps du patrimoine. La dimension mémorielle demeure. Mais elle n'intègre pas seulement les témoignages passés ou ancestraux. Le présent, lui aussi, est à même de témoigner, de transmettre. De même que l'art moderne et contemporain trouve une place au musée, l'Industrie n'est plus seulement reconnue au titre de quelques machines archaïques et obsolètes. Diachronique et synchronique, la patrimonialisation s'affranchit plus volontiers d'un cadre temporel. Ce qui importe désormais, c'est moins "l'âge du patrimoine" que sa capacité à conserver (mémoriser) et transmettre (signifier) un message à l'ensemble de la communauté.

En aucun cas, le statut d'objet patrimonial n'est immanent à la nature de l'objet considéré. "L'objet patrimonial ne se maintient comme tel que pour autant que le groupe social dans les habitudes duquel s'intègre l'objet, l'accepte toujours"'.

Il était inévitable, du fait de son extension sémantique et de sa "popularisation", que le concept de patrimoine intéresse le champ de l'entreprise. Il rejoint la logique entrepreneuriale sur deux points essentiels : le capital et le social. D'une part, la protection et la conservation d'un patrimoine industriel confirme la dimension économique de l'entreprise, entendue comme lieu de capitalisation de biens auxquels est attribuée une valeur. D'autre part, la patrimonialisation affirme la personnalité sociale de l'entreprise, entendue comme espace d'échanges à l'intérieur et à l'extérieur de la sphère productive.

Dans les deux cas, il serait réducteur de considérer le patrimoine comme simple outil de communication commerciale. Il ne constitue pas, à proprement parler, une création ex nihilo d'image et de sens. Dans le cadre de l'entreprise, le patrimoine recouvre des éléments aussi divers que : des infrastructures, des outils de production, des pratiques (par exemple : un savoir-faire, un vocabulaire) propres à un corps de métier, des témoignages publicitaires, des produits... Tout ce qui contribue, en somme, à singulariser l'organisation est potentiellement constitutif d'un patrimoine ${ }^{2}$. C'est pourquoi le patrimoine

1 E. OLlivier, "Les monuments historiques demain...", Terrain, $\mathrm{n}^{\circ}$ 9, oct. 1987, p. 124-127.

2 V. Kollmann et M. Valière, Le patrimoine industriel, Poitiers, Éd. ABCD Mémoire d'image, coll. Libre Accès, 1992. Il y a nécessairement, derrière le concept de patrimoine, une tension entre les principes d'irréductibilité et de spécification. En théorie, le patrimoine n'est pas réductible à une nomenclature de bien matériels ou immatériels. En actes, la patrimonialisation opère une sélection des éléments jugés les plus significatifs d'une identité communautaire. 
d'entreprise n'est pas réductible à quelques objets insignes de l'appareil productif, tels que : le bâti industriel, les infrastructures, les machines et les produits qui matérialisent l'activité économique. D'autres éléments, moins tangibles, ont également valeur patrimoniale. Le geste et la parole du professionnel, l'organisation du travail, le style de management, racontent également l'entreprise et sont susceptibles d'être valorisés.

À la fois matière à communiquer, outil et stratégie de communication, le patrimoine -quel qu'il soit- désigne un "déjà là", une ressource sur laquelle le marketing publicitaire peut s'appuyer, mais qui ne lui appartient pas en propre. Lorsque certaines publicités mentionnent la date de création d'une entreprise ou d'un produit, elles signalent une histoire, une certaine pérennité, indicatives -notamment- de la qualité d'une production. Par cette mention, la publicité travaille l'image de marque. Pour autant, elle ne construit pas l'histoire de l'entreprise. De ce point de vue, la patrimonialisation ${ }^{1}$ échappe, pour partie, au processus de reconnaissance médiatée (l'image construite) dans lequel s'inscrit la communication publicitaire. A cet égard, plusieurs éléments de réflexion méritent d'être soulignés. En premier lieu, on observe que toutes les entreprises ne se prêtent pas avec la même facilité et pour le même bénéfice à une valorisation de leur patrimoine. Celle-ci suppose une histoire, des savoir-faire, une production, un ancrage culturel avérés et dignes d'être valorisés. Par ailleurs, toute patrimonialisation nécessite sa compatibilité avec la politique, les stratégies et les moyens de communication mis en œuvre par l'entreprise (communication globale).

Enfin, la protection et la valorisation d'un patrimoine d'entreprise ne peuvent être strictement motivées par la reconnaissance d'une enseigne, d'une marque ou d'un produit; sans quoi la création publicitaire pourrait, à moindre coût et avec une portée bien supérieure, satisfaire cet objectif.

Pourtant, les industriels semblent aujourd'hui plus enclins à jouer la carte du patrimoine, soucieux de participer à leur tour au mouvement de patrimonialisation qui traverse la société. C'est que, assure Cuisenier,

1 La "patrimonialisation" (néologisme attribué à Pierre Nora) désigne le processus d'appropriation morale des éléments matériels ou immatériels par lesquels une communauté se reconnaît. 
même en matière économique, le patrimoine remplit des fonctions nombreuses, hétérogènes et irréductibles (...) S'opère une véritable valorisation des biens patrimoniaux qui se voient investis d'une signification nouvelle, car à même de procurer un avantage décisif dans la compétition économique ${ }^{1}$.

\section{Formes et les enjeux d'ume patrimonialisation}

Mais de quel(s) avantage(s) décisif(s) parle-t-on ? En quoi le recours au patrimoine est-il potentiellement générateur d'une plusvalue économique? Quels enjeux motivent l'engagement d'un industriel dans la valorisation d'un patrimoine ? Ils sont extrêmement dépendants de la nature du patrimoine considéré et des formes de cette patrimonialisation.

Avant que d'insister sur le caractère institutionnel d'une communication sur le patrimoine, il n'est pas inutile de rappeler les diverses formes d'une valorisation et les objectifs qui leur sont assignés. On peut, de façon schématique, distinguer trois seuils de patrimonialisation auxquels correspondent des réalisations et des objectifs distincts.

Le premier seuil est celui de la conservation d'éléments auxquels est attribuée une valeur informationnelle, historique, esthétique, pécuniaire, symbolique... Il en va des archives industrielles (ex : St. Gobain, Gaz de France, Creusot-Loire, etc.) comme des collections d'entreprises (ex: France Télécom, Christofle, Renault) qui font l'objet d'une conservation, notamment à des fins d'étude et de recherche. L'histoire convoquée -elle n'est pas nécessairement séculaire- répond à un besoin de maitrise, de compréhension des événements, des projets et des créations qui jalonnent le parcours de l'entreprise. Lorsque Renault décide la mise en place d'une cellule "patrimoine et innovation", l'objectif n'est pas seulement archivistique. Il est surtout technologique au sens où l'analyse rétrospective de la firme peut intervenir dans ses productions actuelles et futures. Parce que certains brevets déposés il y a 50 ans ont été précieusement conservés, des solutions techniques ou esthétiques pourront être reprises, à moindre coût, dans la création de nouveaux modèles. Support de réflexion pour l'ingénieur, outil de création pour

1 Jean Cuisenier, cité par Isac CHIVA, "Le patrimoine ethnologique: l'exemple de la France”, Encyclopaedia Universalis, p. 237, Symposium 1990. 
le designer, signe de distinction pour le communicant, les archives et collections constituent des aides précieuses à la décision.

Mais les plans et brevets, dont certains se comptent en kilomètres linéaires ${ }^{1}$, ne sont pas les seules sources d'enseignement pour l'entreprise. Les témoignages d'anciens ingénieurs, techniciens et ouvriers sont des clefs de lecture déterminantes dans l'utilisation ou le renouvellement de techniques devenues illisibles pour les nouvelles générations. L'intérêt récent porté au récit professionnel indique toute la valeur d'une "mémoire vivante", d'un récit ouvrier qui contribuent à la transmission des savoirs et savoir-faire dans l'entreprise ${ }^{2}$.

Il reste que la préservation d'un patrimoine d'entreprise n'est pas toujours à ce point finalisée. Aux fonctions d'étude, de formation, de transmission de connaissances, s'ajoute un objectif plus modeste et marginal de capitalisation de témoignages objectaux dans lesquels l'industriel-collectionneur reproduit son histoire et celle de son entreprise. Certains industriels arguent du lien affectif et identitaire qui les relie à un patrimoine reconstitué en collections. Celles-ci, comme le rappelle Baudrillard, jouent le rôle de miroirs parfaits, au sens où ils ne renvoient pas les images réelles mais les images désirées ${ }^{3}$.

Le second seuil est celui de l'exhibition. Exhibition architecturale, d'abord, où l'entreprise investit visuellement le paysage par la protection ou la valorisation d'un bâti industriel remarquable. La réhabilitation de l'ancienne chocolaterie Menier, à Noisiel (Seine-etMarne), est exemplaire d'une entreprise (Nestlé) qui, visuellement, entend signifier sa présence et son attachement à des valeurs ${ }^{4}$.

Exhibition aussi des collections qui remplissent les conservatoires de techniques et autres académies d'amateurs. Entre le cabinet de curiosité et la foire professionnelle, ces exhibitions publiques

1 Il faut noter, à ce propos, que le patrimoine ainsi capitalisé -constitué ou reconstitué- peut déborder la collecte d'éléments ayant trait à l'entreprise de référence. On sait que les archives industrielles renferment des secrets de fabrication souvent empruntés à des concurrents.

2 J. DeBEsse, "Mémoire d'usine: l'aérospatiale à Châtillon-sous-Bâgneux, l'expérience d'un comité d'établissement", in M. HAMON et F. TORRES (dir.), Mémoire d'avenir: l'Histoire dans l'entreprise, Paris, Economica, 1987, p. 107119.

3 J. BAUdRILLARD, Le système des objets, Paris, Gallimard, 1978, p. 120-121.

4 G. PACAult et J. BERNARD, "Nestlé France à Noisiel. Du patrimoine industriel à la transformation en site tertiaire", L'archéologie industrielle en France, $\mathrm{n}^{\circ}$ hors série, Le patrimoine industriel pour quoi faire?, CILAC, Actes du colloque national de Trégastel des 5, 6 et 7 octobre 1994, juin 1996, p. 25-26. 
contribuent à accroître la notoriété de l'entreprise, en même tant qu'elles renforcent chez les professionnels le sentiment d'appartenir à une corporation.

Enfin, l'ultime étape de la patrimonialisation est celle de l'exposition; forme médiatiquement la plus aboutie, parce qu'elle opère de manière sélective dans le choix de ses publics et des contenus qu'elle présente. L'entreprise -non contente de conserver et de montrer ses richesses- entend contrôler les effets d'une exhibition directe. Musées, expositions et visites de sites industriels constituent ainsi des ensembles sémiotiquement formalisés, organisés et balisés, où la logique de reconnaissance (l'interprétation par le récepteur-visiteur) est anticipée et orientée par la logique de production (encodage par l'émetteur-concepteur). De la présentation, on passe à la représentation : en sélectionnant les signes qu'elle donne à voir et à lire, l'entreprise précise, confirme ou corrige son image publique. Tantôt, la volonté de démontrer une pérennité appelle l'exposition d'outils et d'objets anciens. Tantôt, des mouvements d'opinion ou les inquiétudes de consommateurs plaide pour l'ouverture des locaux et la visite commentée.

Qu'il s'agisse d'affirmer un ancrage local (communication de proximité), de participer à l'action communautaire (communication politique), de défendre une position idéologique (communication militante), de prévenir ou de rassurer (communication de crise)... l'entreprise ne se montre plus seulement. Elle s'explique. On parle, dès lors, d'une communication institutionnelle.

\section{La patrimonialisation comme médiation institutionnelle : signes particuliers d'entreprise}

On perçoit assez facilement en quoi le recours à une histoire, à des symboles est porteur de valeur ajoutée pour l'entreprise. Le patrimoine constitue, en effet, un support de distinction, eu égard au brouillage des messages publicitaires sur des marchés extrêmement concurrentiels et à la saturation des canaux de communication. C'est notamment parce qu'elle ne recourt pas aux moyens et aux canaux de communication classiques que la patrimonialisation se distingue d'une communication publicitaire. Les médias traditionnels (presse, télévision, radio) n'ont ici qu'un rôle de relais. La communication se joue 
ailleurs, au plus proche de l'entreprise, au plus intime et au plus insolite parfois.

La communication institutionnelle, c'est le discours des institutions publiques, privées, associatives sur elles-mêmes. Non pas le discours sur leurs «produits»-ceux qu'elles mettent en œuvre ou qu'elles vendent- mais sur leur nature spécifique, leur légitimité, leurs principes, leur personnalité, leur projet, leurs choix, leurs objectifs, leurs actes, leurs performances ${ }^{1}$.

De tout cela, la patrimonialisation peut rendre compte. Autoréférentielle, cette communication s'écarte, elle aussi, d'une logique produit-marché. Le patrimoine ne constitue pas, à proprement parler, un argument de vente. Il connote plus qu'il ne dénote. C'est pourquoi sa valorisation s'inscrit plus dans la perspective d'une reconnaissance d'ordre affectif que cognitif. La seule exhibition d'objets anciens agrégés en collection ne peut illustrer fidèlement la production d'une entreprise ; pas plus qu'une visite d'atelier automatisé ne permet d'en saisir avec précision le procès industriel. Pourtant, la seule visite d'une exposition ou une brève incursion en milieu professionnel offre déjà une proximité, sinon une intimité, entre l'entreprise et ses hôtes.

Comme toute communication institutionnelle, le patrimoine agit davantage sur l'opinion que sur le marché. Mais il doit, pour cela, être partagé par l'ensemble de la communauté, interne et externe à l'entreprise.

La liaison particulière établie (...) entre une collectivité et son patrimoine peut s'appliquer à des communautés très réduites, à une entreprise, une région, une nation, comme à l'humanité toute entière. La difficulté n'est pas là. Elle est dans la possibilité que des non-membres attachent eux aussi de la valeur aux éléments en question et les considèrent comme faisant partie d'une certaine façon de leur propre patrimoine?

1 "(...)Autocentrée de façon manifeste, quand l'institution parle explicitement d'ellemême, ou de façon indirecte, quand elle s'engage pour une cause étrangère à sa propre finalité" (J. DANIEL, "Communication institutionnelle et d'entreprise", in L. SFEZ (dir.), Dictionnaire critique de la communication, Paris, PUF, t. 2, 1993, p. 1177-1178).

2 J. GADREY, "Gestion patrimoniale, gestion durable des ressources, et évaluation", Politiques et management public, Institut de Management Public, vol, $12, \mathrm{n}^{\circ} 4$, déc. 1994, p. 31-49. 
La montée en puissance du mouvement associatif montre assez que l'attachement au patrimoine industriel n'est pas l'apanage de quelque autorité supérieure ou d'experts en patrimoine. Dans de nombreuses associations, dites corporatistes, le cercle des membres actifs ne cesse d'être élargi aux simples amateurs, quel que soit leur parcours ou leur bagage.

Peu d'indicateurs permettent, à ce jour, d'identifier et d'évaluer une demande sociale en matière de patrimoine à caractère industriel. En revanche plusieurs facteurs encouragent une valorisation, notamment culturelle, de l'entreprise.

En premier lieu, l'affaiblissement des institutions et la perte de légitimité du politique contribueraient à élargir l'espace d'intervention de l'appareil économique. L'espace social, laissé vacant par l'État et les syndicats notamment, serait donc investi par l'industriel. A l'extrême, la reconnaissance de l'entreprise érigée en "modèle" achève le processus de déstabilisation des institutions. Du politique, on attend qu'il se comporte en manager. De l'entreprise publique, on exige rendement et rentabilité. Partout, l'idéologie saint-simonienne semble emporter l'adhésion. Comment interpréter la "libéralisation" de grands services publics (La Poste et France Télécom, par exemple) sinon comme l'affirmation d'un modèle entrepreneurial ?

Par ailleurs, les nouvelles exigences du marché ont bousculé les anciennes organisations paternalistes et tayloriennes. La concurrence exacerbée, la délocalisation des outils de production, l'atomisation du temps de l'information et de la décision, la montée du secteur tertiaire, modifient sensiblement la place et le statut de l'entreprise dans la société.

La dislocation de l'espace usinier, espace jadis physiquement repérable en un lieu, avec une séparation physique entre le "dedans" et le "dehors", entre ce qui était elle et ce qui ne l'était pas, fait place à des formes hybrides d'organisation que les discours managériaux désignent de façon métaphorique: entreprise "projet", entreprise "réseau", entreprise "virtuelle"... .

1 Chr. LE MOËNNE, "Espace public et entreprises: penser la sphère professionnelle", in I. Paillart (dir.), L'espace public et l'emprise de la communication, chap. 3: L'interpénétration de l'espace public et de l'entreprise, Grenoble, ELLUG, 1995, p. 139-162. 
Or cette complexification, cette opacification et cette perte de lien physique entre l'entreprise et son environnement appellent de nouvelles formes de lisibilité et d'intelligibilité de la sphère productive.

Ce mouvement contradictoire d'intégration sociale et de distanciation physique de l'entreprise la conduirait à une gestion stratégique des rapports sociaux dans l'espace de la communication ${ }^{1}$.. Avec une montée alarmante du chômage, la pression sociale et les mouvements d'opinion (groupements écologistes, associations de consommateurs et d'usagers, notamment) focalisent tous les espoirs et tous les soupçons sur l'appareil économique. Pour gérer ou endiguer cette poussée médiatique qui contribue à l'exposer ${ }^{2}$, l'entreprise a dû s'adjoindre les services de nouveaux professionnels : instituts de sondages, relations publiques et autres "dircoms" forment désormais la garde rapprochée des capitaines d'industrie.

Leur mission ne consiste plus seulement à faire connaitre, mais surtout à faire reconnaître publiquement l'entreprise, en affirmant sa place et son rôle dans son environnement politique, social et culturel. De là est né l'espoir (le mythe ?) d'une entreprise "citoyenne" capable d'assumer de nouvelles responsabilités sociétales. Mais n'y aurait-il pas quelque naïveté à considérer cette responsabilisation de l'entreprise dans une perspective altruiste et philanthropique ?

L'impact d'une communication institutionnelle sur des résultats économiques est, certes, difficile à évaluer. Mais la contribution du symbolique et du culturel à une nouvelle médiation entre la sphère productive et la société civile n'en reste pas moins remarquable.

Même la communication publicitaire n'échappe pas à cette orientation institutionnelle. Référentielle, oblique, mythique ou substantielle, la publicité s'est sensiblement diversifiée et complexifiée; au point de rompre définitivement avec sa forme primitive, la réclame ${ }^{3}$. Certaines campagnes publicitaires flirtent avec une stratégie de communication institutionnelle. Les unes manifestent ostensiblement une responsabilité sanitaire et sociale (ex : Leclerc contribuant à la protection de l'environnement par le retrait des sacs plastiques dans ses supermarchés) quand d'autres, parfois sulfureuses (ex : campagnes

1 B. FLORIS, "L'entreprise sous l'angle de l'espace public", in I. PAILLART (dir.), op. cit., p. 119-138.

2 "Exposer": dans la double acception d'ériger en modèle et de mettre en péril.

3 J.-M. FLoCH, Sémiotique, marketing et communication. Sous les signes, les stratégies, Paris, PUF, 1990, p. 183-226. 
supports et les thèmes susceptibles de la représenter. Tantôt, elle retracera la généalogie de ses fondateurs, tantôt elle évoquera ses créations et ses innovations techniques. Historique, technologique, sociologique... l'évocation de l'entreprise peut-être extrêmement polymorphique et polysémique. Elle peut même absorber d'autres communications de l'entreprise en retraçant, par exemple, son parcours publicitaire. Au paroxysme de cette méta-communication, l'entreprise en vient à exposer les affiches et gadgets porteurs d'une identité visuelle et de slogans qui ont fait sa notoriété.

Polymorphique et polysémique, donc, le patrimoine d'entreprise n'est pas nécessairement élitiste dans ce qu'il exhibe et dans les publics auxquels il s'adresse. De l'ouverture de sites en activité à l'aménagement d'espaces muséographiques, les manifestations d'un patrimoine d'entreprise sont aussi nombreuses que variables dans leur forme, leur contenu, leur portée et leur coût. Et les grandes entreprises ne sont pas seules à pouvoir s'exposer et s'expliquer en public. Des entreprises artisanales, même à faible budget, se prêtent facilement à des visites d'ateliers et participent volontiers à des expositions. Certaines ont misé, pour toute promotion, sur la seule détention d'un savoir-faire ou d'une histoire... qui suffisent à les distinguer, à leur assurer notoriété et réputation.

A l'instar des grandes expositions universelles, la présentation -même modeste-d'un patrimoine confirme l'entreprise dans son ancrage national, régional ou local. A la valorisation d'un patrimoine marqué "territorialement", les collectivités ne sont d'ailleurs pas insensibles. Elles encouragent ces exhibitions professionnelles qui contribuent à leur rayonnement et à leur attractivité, notamment touristique. Foires, salons et expositions thématiques participent à l'expression de richesses régionales ou locales, dont l'entreprise reste un producteur et un témoin privilégié.

Enfin, la valorisation d'un patrimoine industriel, à la différence d'autres communications institutionnelles (le sponsoring, notamment), s'inscrit souvent dans la durée. D'une part, et contrairement à l'adage, la valeur d'un patrimoine attend souvent le nombre des années. D'autre part, l'accumulation patiente et sélective d'éléments destinés à être exposés ne laisse guère de place à l'improvisation. Sauf à faire l'objet d'exhibitions ponctuelles, la patrimonialisation de l'entreprise ne constitue pas, à proprement parler, une communication à caractère événementiel. Non réductible aux éléments qui le constituent, un patrimoine survit longtemps à leur disparition ou à leur extinction. 
Sans être nécessairement inaliénable, il porte en substance l'idée d'une pérennité, d'une permanence. Sa fonction éminemment identitaire en limite d'autant le risque d'abandon. Certains industriels l'ont bien compris qui, à l'occasion de rachats ou de fusions, conservent l'enseigne des entreprises acquises ou annexées, par crainte d'en perdre le capital d'image : réputation, ancrage local, etc.

Ce qui est particulier et étonnant dans la protection et la valorisation d'un patrimoine industriel, c'est la référence à une histoire, à des valeurs conservatistes qui, apparemment, sont en contradiction avec l'approche entrepreneuriale, toujours prompte à anticiper et à s'adapter aux changements les plus rapides. Mais, c'est précisément face à une telle instabilité de son environnement et de ses conditions d'exercice que l'entreprise éprouve le besoin de temporiser, d'affirmer une pérennité et une stabilité, de rassurer tous ceux qui s'inquiètent de son devenir: ses actionnaires, ses salariés, ses partenaires, ses clients... Il faut comprendre que la référence à des acquis, à des valeurs, à des savoirs n'est en rien contradictoire avec une politique ambitieuse et dynamique ${ }^{1}$. Si l'entreprise s'appuie sur des références patrimoniales, c'est qu'elle mesure les enjeux que recouvrent un savoir-faire, une créativité, une originalité maintes fois éprouvés et dont elle a tout lieu d'être fière. A charge pour elle de ne pas hypothéquer ce capital confiance, mais de l'enrichir au contraire en se projetant, sans attendre, vers l'avenir.

\section{La patrimonialisation : miroir ou prisme de l'entreprise?}

La patrimonialisation de l'entreprise, comme médiation institutionnelle à caractère culturel, participe indéniablement à la conjonction de l'espace privé professionnel et de l'espace public. Certains analystes parlent d'une inclusion de l'un dans l'autre ${ }^{2}$. Les plus optimistes s'en réjouissent; considérant que l'appareil industriel gagne en visibilité et en sociabilité en se faisant mieux connaître ${ }^{3}$. Les plus

1 F. TORRES, "Retour vers l'avenir: l'Histoire dans l'entreprise", in M. HAMON et F. TORRES, op. cit., p. 21-39.

2 Chr. LE MOËNNE, op, cit; ; B. FLORIS, op. cit.

3 N. D'ALMEIDA, L'entreprise à responsabilité illimitée. La citoyenneté d'entreprise en questions, Paris, Liaisons, Communication-Innovation, 1996. 
critiques s'inquiètent, au contraire, d'une tendance hégémonique de l'entreprise à qui seraient abandonnées les clefs de la cité.

A ce débat, la question d'une patrimonialisation n'apporte qu'un éclairage modeste et nous invite à la plus grande prudence; notamment parce que la problématique d'une interpénétration de l'espace public et de l'entreprise ne se limite pas à l'analyse des rapports de médiation entre le dedans et le dehors, entre la sphère productive et la société civile ${ }^{1}$. En cela, notre propos mériterait un développement sur les effets d'une patrimonialisation dans l'organisation de l'entreprise et de ses rapports sociaux internes.

A défaut, nous nous limiterons à conclure sur le caractère ambivalent d'une patrimonialisation où s'affrontent deux systèmes sémiotiques. Le premier renvoie à ce qui est montré effectivement. Le second réfère à la volonté de construire un dispositif sémiotique vraisemblable, mais artificiel. Car l'entreprise a beau exciper de son authenticité vraie, elle n'en demeure pas moins sélective dans ce qu'elle donne à voir et à interpréter. Par l'ouverture de l'espace usinier aux visiteurs curieux ou la création de dispositifs dévolus à l'accueil non marchand, l'entreprise fait acte de sociabilité. Elle se montre et s'explique à l'attention de publics auxquels elle ne reconnaissait naguère qu'un statut de clients ou d'usagers.

Pour autant, ces effets d'exhibition directe ou ces mises en scène de l'appareil industriel suffisent-ils à révéler l'entreprise ? Entre le simulacre et son double, l'entreprise ne propose nécessairement qu'une représentation ${ }^{2}$ de ce qu'elle est effectivement. Il est bien évident que l'entreprise ne nous dit pas tout et qu'elle ne peut pas tout nous dire.

Il faut en tout cas se garder de surestimer l'engagement de l'entreprise en faveur de son patrimoine et de sa valorisation. Car cette nouvelle conscience patrimoniale n'est encore le fait que d'une

1 B. MiEge, "L'espace public: perpétué, élargi et fragmenté", in I. PAILLART, op. cit. B. FLORIS, op, cit.

2 Nathalie Heinich rappelle cette relation intersubjective qui fonde la représentation: "Ce qui définit le mieux la représentation, c'est la dialectique, le jeu entre l'absence et la présence: par où un objet absent est rendu, «pour ainsi dire», présent. Et, c'est bien sûr, dans ce pour ainsi dire que se loge toute l'ambiguïté et toute la gamme de validité d'une représentation, en tant qu'elle peut se trouver créditée ou au contraire, sujette à discréditation (...)" (N. HEINICH, "Représentation", in L. SFEZ (dir.), op. cit., vol. 1, p. 37-38). 
poignée d'entreprises en Francel. Constatons que la visite d'entreprise, forme la plus élémentaire d'ouverture à la culture, n'est pas encore une pratique généralisée à l'ensemble des secteurs et branches d'activité économiques. Prudence ou méconnaissance des effets d'une communication à caractère culturel ? Les filières dites "sensibles", toujours soucieuses de leur confidentialité, demeurent ainsi des lieux fermés aux visiteurs. D'autres entreprises prétextent le manque d'attrait ou l'immatérialité de leur production. Certaines firmes craignent de porter préjudice à une image de marque longuement construite, à force de slogans commerciaux, de jingles publicitaires et de papier glacé. Elles ont peur de ne pas correspondre aux représentations qu'elles ont créées et nourries chez le consommateur, par le recours massif à d'autres médias.

Parfois, lorsque la visite est autorisée, les contraintes d'hygiène et de sécurité sont telles qu'elles imposent une mise à distance du visiteur. Alors, des passerelles ont été aménagées. Des aires de repos, à l'écart des machines, protègent les visiteurs du bruit, des projections, des étincelles. Mais en sélectionnant les passages, les lieux où l'on circule et ceux où l'on stationne, on s'écarte aussi des espaces plus anonymes, innommables ou interdits. Le parcours de visite a souvent pour effet d'atomiser l'appareil industriel ou de l'aseptiser. Ce couloir vitré qui surplombe les postes de travail d'une coopérative laitière permet, certes, de visualiser les étapes successives de la pasteurisation et du conditionnement. Mais le regard se perd dans un dédale de cuves et de tuyauteries chromées, sans que jamais ne filtre un son, une odeur, ni même ne se voit une goutte du produit que cette formidable machinerie stérilise et homogénéise. Aussi, la visite pose le problème

1 Par indifférence, par crainte ou par nécessité, seulement $20 \%$ des entreprises françaises s'ouvrent à la visite (sondage IFOP, 1990). Pour expliquer ce mutisme, cette réticence, ou cette sélection, Desvallées convoque ce qu'il nomme le "complexe du sale" (A. DesvalléEs, "Musées scientifiques, musées techniques, musées industriels", in Br. Schroeder-Gudehus (dir.), La société industrielle et ses musées: demande sociale et choix politiques, 1890-1900, Éd. des Archives contemporaines, 1992, p. 97-107; J.-M. STAUDENMAIER, "Expositions «propres», expositions «sales»: l'esthétique technologique d'Henry Ford", in Br. SchroederGudehus, op. cit., p. 171-191; D. JACOBI et Ph. GuILlET, "Quels musées pour la moutarde de Dijon?", Actes du colloque CISTE de Nice Sophia-Antipolis, Z'Éditions, 1993, p. 83-90). Un complexe qui se dédouble: d'une part, l'industriel préfère montrer le beau plutôt que le produit vulgaire; d'autre part, il ne tient pas à parler de ses méthodes et s'impose un devoir de réserve en invoquant un secret professionnel. 
d'une visibilité, sans laquelle l'intelligibilité de l'entreprise apparaît fortement contrariée 1 .

Tout cela illustre combien l'industriel, malgré le caractère instantané, participatif d'une communication en direct, sait se montrer sélectif dans sa production de signes. D'ailleurs, par ce qu'elle exhibe ostensiblement, l'entreprise ne cache-telle pas plus efficacement encore une partie d'elle-même?

Le musée constitue, à cet égard, une forme extrêmement élaborée de mise en scène. Là où la visite pouvait encore trahir les aspects les plus authentiques de son activité, le musée instaure véritablement une clôture sémiotique autour de son patrimoine. Par lui, l'entreprise procède à la reconstitution de son histoire, de son parcours économique, de ses choix politiques et stratégiques. Dans le même temps, cet espace est un lieu de simulation dès lors qu'il extrait l'objet industriel de son contexte. L'entreprise prélève sa substance et, qu'elle le veuille ou non, la dénature en lui conférant un statut culturel. Ainsi l'entreprise est-elle souvent présentée comme un système cohérent, dans une évocation esthétisante et linéaire, qui tend à gommer le prosaïsme d'une production, les accidents de l'histoire, les éventuels dysfonctionnements.

Dans ces conditions, que vaut le pari de l'entreprise comme objet ou acteur d'une communication culturelle? En ces lieux sémiotiquement organisés, codifiés, contrôlés... une interprétation libre de l'entreprise est-elle encore de mise ? L'entreprise, de forteresse secrète et infranchissable est devenue un lieu de médiation culturelle ${ }^{2}$. Il faut s'en féliciter, en mesurant le chemin parcouru. Mais il faut également garder à l'esprit que l'entreprise, par nature, est un lieu de production à vocation économique. Et que rien, pas même la quête de reconnaissance, ne saurait la détourner de cette vocation.

I Y. JEANNERET, "La visite d'entreprise: une situation de communication complexe", Communications et langages, Paris, 1er sem, 1991, p. 93-105.

2 A. MichalowsKa, "Le tourisme industriel, l'entreprise se montre de l'intérieur comme un musée vivant...", Le culte de l'Entreprise: mutations, valeurs, cultures, Autrement, $\mathrm{n}^{\circ}$ 100, sept. 1988, p. 105-109. 